\title{
Development and Characterization of Niosomal Formulations of Doxorubicin Aimed at Brain Targeting
}

\author{
Marco Bragagni ${ }^{\mathrm{a}}$, Natascia Mennini ${ }^{\mathrm{a}}$, Carla Ghelardini ${ }^{\mathrm{b}}$, Paola Mura $^{\mathrm{a}}$ \\ ${ }^{a}$ Department of Pharmaceutical Sciences, University of Florence, Florence, Italy. \\ ${ }^{b}$ Department of Preclinical and Clinical Pharmacology, University of Florence, Florence, Italy. \\ Received, December 14, 2011; Revised, January 5, 2012; Accepted, January 23, 2012; Published, January 25, 2012
}

\begin{abstract}
Purpose. The aim of the present work was the development and characterization of a niosomal formulation functionalized with the glucose-derivative N-palmitoylglucosamine (NPG) to obtain a potential brain targeted delivery system for the anticancer agent doxorubicin. Methods. Five different methods have been examined for vesicle preparation. Light scattering and transmission electron microscopy were used for vesicle characterization, in terms of mean size, homogeneity and Zeta potential, and selection of the best composition and preparation conditions for developing NPG-functionalized niosomes. Drug entrapment efficiency was determined after separation of loaded from unloaded drug by size exclusion chromatography or dialysis. Preliminary in vivo studies were performed on rats, injected i.v. with $12 \mathrm{mg} / \mathrm{kg}$ of doxorubicin as commercial solution (Ebewe, $2 \mathrm{mg} / \mathrm{mL}$ ) or NPG-niosomal formulation. Drug amounts in the blood and in the major organs of the animals, sacrificed 60 min post injection, were determined by HPLC. Results. The selected formulation consisted in Span:cholesterol:Solulan:NPG (50:40:10:10 mol ratio) vesicles obtained by thin-layer evaporation, leading to homogeneous vesicles of less than $200 \mathrm{~nm}$ diameter. This formulation was used for preparation of NPG-niosomes loaded with doxorubicin (mean size $161 \pm 4 \mathrm{~nm}$, encapsulation efficacy $57.8 \pm 1.8 \%)$. No significant changes $(\mathrm{P}>0.05)$ in vesicle dimensions, Zeta potential or entrapment efficiency were observed after six months storage at room temperature, indicative of good stability. I.v. administration to rats of the NPG-niosomal formulation allowed for reducing drug accumulation in the heart and keeping it longer in the blood circulation with respect to the commercial formulation. Moreover, a doxorubicin brain concentration of $2.9 \pm 0.4 \mu \mathrm{g} / \mathrm{g}$ was achieved after $60 \mathrm{~min}$, while the commercial solution yielded undetectable drug brain concentrations $(<0.1 \mu \mathrm{g} / \mathrm{g})$. Conclusions. The developed NPG-niosomal formulation gave rise to stable, nano-sized vesicles, able to improve doxorubicin brain delivery. Positive results of preliminary in vivo studies require future pharmacokinetic studies to gain more insight into the mechanism of drug transport of functionalized niosomes.
\end{abstract}

This article is open to POST-PUBLICATION REVIEW. Registered readers (see "For Readers") may comment by clicking on ABSTRACT on the issue's contents page.

\section{INTRODUCTION}

The development of suitable strategies for effective target delivery of drugs to brain is a very active and challenging research field, since the same mechanisms that protect the brain against potentially toxic substances, also strongly restrict the entry of many potential therapeutic agents. The blood-brain barrier (BBB) is a dynamic and complex structure located at the level of endothelial cells of the cerebral capillaries, which acts as a physical and metabolic barrier, preventing the passage of several kinds of therapeutic agents, including antibiotics, antitumourals and neuropeptides from the bloodstream to the central nervous system. Therefore, it represents a significant obstacle in the effective pharmacological treatment of central nervous system (CNS) disorders. In particular, great efforts are presently focused on improving the CNS bioavailability of drugs used for brain cancer therapy. In fact, the treatment of numerous brain tumours is particularly difficult, due to several factors, such as impossibility of completely removing the diseased tissue by surgical intervention, tendency to recurrence after first-line therapy, poor response to first and/or second-line therapy (1).

Several approaches have been explored to improve therapeutic agent penetration through the $\mathrm{BBB}$, including disruption of the BBB integrity, or bypassing the BBB (by intracerebroventricular, intracerebral or intrathecal drug infusion), or use of lipid-soluble prodrugs.

Corresponding Author: Paola Mura, Department of Pharmaceutical Sciences, University of Florence, Florence, Italy: E-mail: paola.mura@unifi.it 
However, the first two procedures are very invasive and can cause high incidence of hemorrhage, cerebrospinal fluid leak, neurotoxicity, and central nervous system infection (2). On the other hand, the use of prodrugs often is not possible and, moreover, it suffers from important limitations, such as poor selectivity, poor retention, possibility for reactive metabolites, which all concur to decrease the drug therapeutic index (3). The use of colloidal carrier systems, such as liposomes or nanoparticles, represents an interesting alternative to the above strategies for transporting drugs across the BBB $(4,5)$.

Among the different kinds of colloidal systems, non ionic surfactant vesicles (niosomes) are presently studied as novel and efficient drug carriers, offering a number of advantages with respect to classic liposomes, such as higher chemical stability, greater ease of production, lower cost, and wider formulation versatility (68). Moreover, the vesicle performance can be further improved by chemical modification, using suitable ligands coupled to their surface. Niosomes functionalized with glucose analogues have been suggested as a tool to deliver drugs to the brain, considering the high level of cerebral glucose uptake (9). In particular, the derivatized surfactant N-palmitoylglucosamine (NPG) has been successfully employed for brain targeting of the vasoactive intestinal peptide (10). Although the exact mechanism of action of NPG-bearing niosomes has yet to be clarified, recognition of glucosamine exposed on the surface of the vesicles by the glucose transporter GLUT-1, highly expressed on the BBB cells, has been hypothesized (10).

Doxorubicin is considered one of the most potent and versatile anticancer agents against numerous malignant tumours (11). Unfortunately its therapeutic potential is limited by its low ability to cross the BBB (1). Therefore, the development of a strategy allowing doxorubicin delivery to the brain is of primary importance and would offer the possibility to add this highly active antitumoural agent to the list of drugs available for the therapy of brain tumours. Different colloidal carrier formulations of doxorubicin have been developed, mainly aimed to improve its antitumor activity and decrease its cardiotoxicity (12-19). Among these, poly(butylcyanoacrylate) nanoparticles coated with polysorbate80 enabled a significant enhancement of brain concentration of doxorubicin in rats (18). Moreover, quaternary ammonium- $\beta$-cyclodextrin nanoparticles improved doxorubicin permeability across the BBB in vitro (19). However, at the best of our knowledge, no attempts have been made of exploiting functionalized niosomes as carriers to improve the delivery of doxorubicin into brain areas. In this regard, the use of glucose-bearing niosomes loaded with doxorubicin could represent a novel useful tool for drug active targeting to brain tumours, further supported by the fact that GLUT-1 receptors are over expressed in solid tumours $(20,21)$.

According to these premises, the aim of the present work was the development of an effective niosomal formulation based on sorbitan esters (Span) as main surfactant and bearing NPG, in view of its possible application in brain targeted delivery of doxorubicin.

Since it is known that the self-assembly of surfactants into niosomes is a complex phenomenon which can be influenced by both the nature of surfactants, the presence of membrane additives and the preparation method $(13,22)$, in the first part of the project, the effect of different formulation and process variables on niosome size and polydispersity was investigated by dynamic light scattering and transmission electron microscopy analyses, to individuate the best type and concentration of components and the most suitable preparation conditions for obtaining stable and homogeneous vesicles of nano-sized dimensions. The results of this initial screening were used for a rational selection of the best niosomal formulation, which was then functionalized with NPG. Preparation conditions of NPG-bearing niosomes were optimized in terms of morphological properties, sizing and homogeneity, before preparation of doxorubicinloaded-NPG-niosomes. The drug-loaded formulation was characterized for morphological aspect, particle size, Zeta potential, encapsulation efficacy and stability after six months storage at room temperature. Moreover it was i.v. administered to rats for a preliminary evaluation of its actual ability to target the drug to the brain.

\section{MATERIALS AND METHODS}

\section{Materials}

Solulan C24 (Poly-24-oxyethylene cholesteryl ether, SOL) was kindly donated by Lubrizol (Cleveland, Ohio, USA). Doxorubicin hydrochloride (DOX) injectable solution (Ebewe, $2 \mathrm{mg} / \mathrm{mL}$ ) was provided by Sandoz Spa (Roma, Italy). Cholesterol (CHL), sorbitan monopalmitate 
(Span 40, HLB 9.8), sorbitan stearate (Span 60, HLB 4.7), sorbitol, palmitic acid Nhydroxysuccinimide and glucosamine were provided by Sigma-Aldrich (Milan, Italy). Phosphate buffer solution $\mathrm{pH}$ 7.4, 0.1 M (PBS) was prepared according to Eur. Pharm. $6^{\text {th }}$ Ed.. Distilled water was used throughout the study. All other reagents and solvents were from Sigma Aldrich (Milan, Italy).

\section{Synthesis of N-palmitoyl glucosamine (NPG)}

The glucose-derivatized surfactant Npalmitoylglucosamine (NPG) was synthesized according to the method of Dufes et al. (9) slightly modified. Briefly, glucosamine $(1.27 \mathrm{~g})$ was added to $1.5 \mathrm{~mL}$ of triethanolamine and 220 $\mathrm{mL}$ of DMSO, and the mixture stirred $30 \mathrm{~min}$ at room temperature. The palmitic acid $\mathrm{N}$ hydroxysuccinimide $(2.5 \mathrm{~g})$ dissolved in $\mathrm{CHCl}_{3}$ was then added to the mixture, left stirring $72 \mathrm{~h}$ at room temperature, under nitrogen atmosphere, protected from the light. Immersion in an ice bath and $100 \mathrm{~mL}$ addition of cold water favoured complete precipitation of the product, which was collected by filtration, washed with water, DMSO and ethanol and then dried at $40^{\circ} \mathrm{C}$ for $48 \mathrm{~h}$. It was stored at $4^{\circ} \mathrm{C}$ protected from the light. The product was characterized by ${ }^{1} \mathrm{H}$ NMR (Bruker Avance 400 (Milan, Italy), deuterated DMSO solvent, $5 \mathrm{~mm}$ tube, $64 \mathrm{~K}$ time domain size, 4000 $\mathrm{Hz}$ spectral width, $8.2 \mu \mathrm{s}$ pulse width, $8.18 \mathrm{~s}$ acquisition time, $1 \mathrm{~s}$ relaxation delay), ESI-MS (Finnigan LTQ (Milan, Italy), 5KV spray voltage, $45 \mathrm{~V}$ capillary tube voltage, $275^{\circ} \mathrm{C}$ capillary tube temperature, positive ion model), and DSC analysis (Mettler TA 4000, $\mathrm{STAR}^{\mathrm{e}}$ system (Mettler-Toledo, Greifensee, Switzerland), $10^{\circ}$ $\mathrm{C} / \mathrm{min}$, range $30-300^{\circ} \mathrm{C}$ ).

\section{Preparation of niosomes}

Niosomal suspensions were prepared by five different methods and Span 40 and Span 60 were tested as main surfactant. CHL and SOL were also included in the niosomal formulations in different molar ratios. The total concentration of components ranged from 0.1 to $38 \mathrm{mM}$.

1) Thin layer evaporation (TLE)-vortex method: the surfactant or surfactant-lipid mixture was dissolved in $\mathrm{CHCl}_{3}$ and introduced in a round-bottom flask; the solvent was removed under vacuum to form a thin layer on the flask wall. After hydration of the thin layer with $5 \mathrm{~mL}$ of PBS, 4 cycles of heating ( $3 \mathrm{~min}$ at $65^{\circ} \mathrm{C}$ ) and vortex mixing $(3 \mathrm{~min})$ were performed. The temperature of $65^{\circ} \mathrm{C}$ was selected since it was above the phase transition temperature of Span 60, which was the component of the lipid mixture with the highest value of transition temperature $\left(50^{\circ}\right.$ C) (23).

2) Thin layer evaporation (TLE)-paddle stirring method: the surfactant or surfactant-lipid thin layer was obtained as above and hydrated with $25 \mathrm{~mL}$ of PBS. A paddle was then introduced in the flask and the suspension stirred $30 \mathrm{~min}$ at $65^{\circ} \mathrm{C}$.

3) Reverse phase evaporation method: $10 \mathrm{~mL}$ of surfactant or surfactant-lipid mixture dissolved in $\mathrm{CHCl}_{3}$ were added to $5 \mathrm{~mL}$ of PBS; the resulted dispersion was sonicated 3 $\mathrm{h}$ (Eurosonic 44 ultrasonic bath, Anwendungstechnik, Offenbach, Germany) to form a W/O emulsion. $\mathrm{CHCl}_{3}$ was then removed by rotary evaporation.

4) Proniosome method: Sorbitol was ground 30 $\min$ at $24 \mathrm{~Hz}$ in a high-energy vibrational micromill (Retsch GmbH, Haan, Germany); the $150-300 \mu \mathrm{m}$ granulometric fraction was isolated (analysette AS200 digit, Retsch $\mathrm{GmbH}$, Haan, Germany) and $1 \mathrm{~g}$ was added to a round-bottom flask connected to a rotary evaporator. The surfactant or surfactant-lipid mixture dissolved in $\mathrm{CHCl}_{3}(3 \mathrm{~mL})$ was introduced onto the powder surface by sequential spraying, alternated to solvent evaporation. After addition of the last aliquot, the solvent was completely removed obtaining "proniosomes" (24). Niosomes were obtained by hydrating proniosomes with 20 $\mathrm{mL}$ of PBS.

5) Nitrogen bubbling method: the surfactant or surfactant-lipid mixture was suspended in 20 $\mathrm{mL}$ of PBS in a round-bottom flask. A probe providing a nitrogen gas flux was introduced and the suspension left bubbling $20 \mathrm{~min}$ at $70^{\circ} \mathrm{C}(22)$.

After preparation, according to the different examined techniques, all the niosomal suspensions were subjected to treatments for size reduction, as described in the following section.

\section{Reduction of size of niosomes}

Two different methods commonly used for size reduction of niosomes have been evaluated and compared. With this aim, all the niosomal suspensions (obtained according to the above described methods) were centrifuged $15 \mathrm{~min}$ at $4000 \mathrm{rpm}$ (Hermle Z200A compact centrifuge, 
Labortechnik GmbH, Wehingen, Germany), and the supernatant collected and divided in two portions: one was sonicated (Sonopuls HD 2200, $200 \mathrm{~W}$ power, probe MS 72, Bandelin Electronic $\mathrm{GmbH}$, Berlin, Germany) for 5 min with the instrument set at $60 \%$ of its maximum power; the other was extruded (Extrusor LiposoFast-Basic, Avestin Europe GmbH, Mannheim, Germany) 11 times through nitrocellulose membranes (Nucleopore $100 \mathrm{~nm}$, Whatman, Dassel, Germany). All the samples were stored at $4^{\circ} \mathrm{C}$ protected from the light.

\section{Preparation of NPG-niosomes and doxorubicin loaded-NPG-niosomes}

Based on the results of the previous screening, the total concentration of components chosen for the preparation of NPG-niosomes was $38 \mathrm{mM}$ and they were composed by Span:CHL:SOL:NPG (50:40:10:10 mol ratio). The TLE-paddle and TLE-vortex (both performed at 65 and $90^{\circ} \mathrm{C}$ ) were selected as preparation methods. After preparation, all suspensions were centrifuged and sonicated as described above. To assess the applicability of these techniques for drug-loaded vesicles preparation, the stability of DOX in these experimental conditions was assessed, maintaining the drug solution under stirring $1 \mathrm{~h}$ at $65^{\circ} \mathrm{C}$ and $90^{\circ} \mathrm{C}$, protected from the light. The residual unaltered drug was then quantified by HPLC analysis.

Drug-loaded NPG-niosomes were prepared according to the TLE-paddle method, using the same concentrations and component ratios previously used for empty NPG-niosomes. The aqueous phase consisted of $20 \mathrm{~mL}$ DOX solution $(2 \mathrm{mg} / \mathrm{mL})$.

\section{Characterization of niosomal suspensions}

Particle size and Zeta potential of vesicles were determined by dynamic light scattering using a Zetasizer Nano ZS90 (Malvern Instruments, Malvern, UK). Niosomal suspensions were properly diluted with distilled water to avoid multiscattering phenomena. For particle size measurements, six independent samples were taken from each dispersion, and measured at $25 \pm 0.1^{\circ}$ C. An average dimensional distribution was then calculated referring to the mode (i.e. the most frequently occurring value), which is the value which best approximates the mean diameter of the vesicles. The particle size distribution was also characterized by means of the polydispersity index (PDI). For Zeta potential determination, the niosomal suspensions were suitably diluted with distilled water and dropped into the Zetasizer electrophoretic cell. Each sample was measured six times at $25 \pm 0.1^{\circ} \mathrm{C}$. The effective formation of the vesicles was investigated by transmission electron microscopy (TEM) analysis (Philips CM12 TEM). A drop of the dispersion was directly placed onto a formvar membrane coated grid and stained by adding a drop of $2 \%(\mathrm{w} / \mathrm{w})$ uranyl acetate solution, removing the excess solution using a filter paper and followed by a through air-drying.

\section{Drug encapsulation efficiency}

Encapsulation efficiency was indirectly determined by using the size exclusion chromatography and dialysis methods, which both allowed separation of the unloaded drug from niosomes (25-28).

According to the size exclusion chromatography method, $0.5 \mathrm{~mL}$ of each sample of DOX-NPG-niosomal dispersion was loaded onto a Sephadex G50 column (granulometry 20$80 \mu \mathrm{m})$ and eluted with water. Loaded-niosomes eluted as a pale pink liquid, while free DOX (redcoloured) remained bound to the column. Vesicles were disrupted through addition of $0.05 \mathrm{~mL}$ TritonX-100 followed by ultra-centrifugation at $120000 \mathrm{~g}$ for $1 \mathrm{~h}$ (Beckman L8-M, Beckman Coulter s.r.l., Milan Italy). The total drug amount in the niosomal dispersion and the drug amount actually encapsulated were obtained by HPLC assay of DOX in the supernatant after disrupting the vesicles before and after gel chromatography, respectively. The encapsulation efficiency was calculated according to the following equation:

$$
\mathrm{EE} \%=\frac{[\text { encaps. drug }]}{[\text { total drug }]} .100
$$

All the data are the mean of five separate experiments.

According to the dialysis method, $3 \mathrm{~mL}$ of DOX-NPG-niosomal dispersion was dropped into a cellulose acetate dialysis bag (Spectra/Por ${ }^{\circledR}$, MW cut-off 12000, Spectrum, Canada) immersed in $150 \mathrm{~mL}$ of distilled water and magnetically stirred at $30 \mathrm{rpm}$. Unencapsulated drug was separated through exchange of receiving medium every $30 \mathrm{~min}$, until the level of dialysed drug become undetectable. The amount of diffused drug was determined by HPLC analysis of the collected solutions. The total amount of drug initially present in each niosomal suspension was determined by TritonX-100 addition followed by 
ultra-centrifugation (as described above) of $1 \mathrm{~mL}$ of non-dialyzed sample (to disrupt the vesicles) followed by DOX assay in the supernatant by HPLC. The percent of encapsulation efficiency (EE\%) was determined by the following equation:

$$
\mathrm{EE} \%=\frac{[\text { total drug }]-[\text { diffused drug }]}{[\text { total drug }]} .100
$$

Each result is the mean of three separate experiments.

\section{Stability studies of niosomal dispersions}

Stability studies of drug-loaded and unloaded niosomal dispersions were carried out by storing the samples at $4^{\circ} \mathrm{C}$ and $25^{\circ} \mathrm{C}$, protected by the light and sealed. Particle size, Zeta potential, PDI and encapsulation efficiency (determined after separation of loaded from un-loaded drug by size exclusion chromatography) were measured immediately after the preparation and every 15 days for six months.

\section{Animal testing}

Experiments were performed in healthy Sprague Dawley rats of 360-390 g (Harlan Laboratories, Udine, Italy). In vivo experiments were carried out in accordance with the Guide for Care and Use of Laboratory Animals as adopted and promulgated by the Italian National Institute of Health and according to the EC Directive 86/609/EEC for animal experiments. The Florence University's Animal Ethics Committee approved the protocol. All efforts were made to minimize animal suffering and to limit the number of animals used. The rats, maintained under controlled lighting (12 h light-dark cycle) and temperature $\left(23^{\circ} \mathrm{C}\right)$, had ad libitum access to food and water. Animals were divided into two groups of six. The first group received the commercial doxorubicin solution and the second group received the NPG-niosomal formulation. In both groups, the formulations were injected i.v. in the tail vein in a dose of $12 \mathrm{mg} \mathrm{DOX} / \mathrm{kg}$. After 60 min post injection, the animals were anesthetized and sacrificed. The blood was collected and the major organs were removed and weighed. Plasma was separated from blood by centrifugation at $3000 \times \mathrm{g}$ for $20 \mathrm{~min}$ and the organs were frozen until assay. Solid tissues were homogenized in PBS solution. Samples $(450 \mu \mathrm{L})$ of homogenised tissues or plasma were added with daunorubicin as the internal standard and then extracted into an acetonitrile-ammonium acetate mixture (1.5:1.0 $\mathrm{v} / \mathrm{v})$. After 5 min sonication in an ice bath, each sample was centrifuged at $6000 \mathrm{x} g$ for $10 \mathrm{~min}$. The supernatant was transferred to a new tube. This step was repeated 3 times, by adding $2 \mathrm{~mL}$ of fresh solvent for each extraction. The supernatants were collected and evaporated to dryness under a nitrogen gas stream. The residue was reconstituted with $2 \mathrm{~mL}$ of acetonitrile/water/phosphoric acid $(100 \mathrm{mM})$ 28:47:25 (v/v) and analyzed by HPLC as described below.

\section{HPLC analysis of doxorubicin}

Quantitative assay of DOX was carried out by a high-performance liquid chromatograph (HP-120 Agilent Technologies, CA, USA) equipped with a UV-VIS detector (Shimadzu, Kyoto, Japan) according to the method of Cielecka-Piontek et al. (29), which was slightly modified. A Hibar RPC18 column, $5 \mu \mathrm{m}$ particle size, $150 \mathrm{~mm}$ x $46 \mathrm{~mm}$ (Merck, Darmstadt, Germany) was used as the stationary phase. The mobile phase was a 45:55 $\mathrm{v} / \mathrm{v}$ mixture of acetonitrile:sodium laurilsulfate acidic aqueous solution (consisting of $2.88 \mathrm{~g} / 1$ of sodium laurilsulfate and $2.25 \mathrm{~g} / \mathrm{l}$ of phosphoric acid); the flow rate was $1 \mathrm{~mL} / \mathrm{min}$. UV detection was carried out at $254 \mathrm{~nm}$. The limit of the spectrophotometric detection was $1 \mu \mathrm{g} / \mathrm{mL}$.

For DOX assay in plasma and organs, the mobile phase was changed to acetonitrile/water/phosphoric acid $(100 \mathrm{mM})$ 28:47:25 (v/v), and a fluorescence detector (G1321A, Agilent Technologies, CA, USA) was used. Analyses were performed at an excitation wavelength of $475 \mathrm{~nm}$ and an emission wavelength of $580 \mathrm{~nm}$. The detection limit of the fluorimetric detection was $0.1 \mu \mathrm{g} / \mathrm{mL}$.

\section{STATISTICAL ANALYSIS}

Results of niosomal characterization analyses (vesicle size, Zeta potential, PDI and drug encapsulation efficiency) and of in vivo experiments were statistically analyzed by ANOVA (one-way analysis of variance) followed by the Student-Neuwman Keuls multiple comparison post test (GraphPad Prism 4 software Inc, San Diego, CA, USA). The differences were considered statistically significant when $\mathrm{P}<0.05$.

\section{RESULTS}

Synthesis of N-palmitoyl glucosamine (NPG) Proton assignments by ${ }^{1} \mathrm{H}$ NMR of NPG were the following: $\delta 0.86 \mathrm{ppm}=\mathrm{CH}_{3}$ (palmitoyl), $\delta 1.24$ 
ppm $=\mathrm{CH}_{2}$ (palmitoyl), $\delta 1.89 \mathrm{ppm}=\mathrm{CH}_{2}$ (palmitoyl shielded by carbonyl), $\delta 2.09 \mathrm{ppm}=$ $\mathrm{CH}_{2}$ (adjacent to carbonyl protons), $\delta 3.01 \mathrm{ppm}$ $=\mathrm{CH}(\mathrm{C} 2$ sugar proton), $\delta 3.3-4.0 \mathrm{ppm}=$ nonexchangeable sugar protons. Mass spectrometry data yielded one main peak corresponding to 440 $\left(100 \%[\mathrm{M}+\mathrm{Na}]^{+}\right)$and further minor peaks 418 $\left(26.5 \%, \mathrm{M}^{+}\right), 857\left(25 \%,[2 \mathrm{M}+\mathrm{Na}]^{+}\right), 456(15 \%$, $\left.[\mathrm{M}+\mathrm{K}]^{+}\right)$and $400\left(15 \%,\left[\mathrm{M}+\mathrm{H}-\mathrm{H}_{2} \mathrm{O}\right]^{+}\right)$. DSC analysis showed a single endothermic peak at $212^{\circ} \mathrm{C}$ ( $\mathrm{T}_{\text {onset }} 118^{\circ} \mathrm{C}, \mathrm{T}_{\text {endset }} 223^{\circ} \mathrm{C}$ ). All these findings indicated that NPG was successfully obtained.

\section{Selection of the best preparation method and formulation for niosome preparation}

Sonication was always more effective than extrusion in reducing the vesicle dimensions of niosomal dispersions. Probe sonication $(5 \mathrm{~min}$, power $60 \%$ ) of all the prepared niosomal dispersions enabled a reduction of the vesicles mean diameter of about $30 \%$, irrespective of the preparation method. On the contrary, extrusion for eleven times through $100 \mathrm{~nm}$ pore size nitrocellulose membranes did not significantly modify the vesicle size.

Among the examined preparation techniques, the "nitrogen bubbling" method had the advantage, with respect to all the other ones, of avoiding the use of toxic organic solvents. However, unfortunately, for all the component combinations and concentrations tested, the vesicles obtained with this method were not homogeneous (PDI $>0.46$ ) and particularly large, with a mean diameter higher than $430 \mathrm{~nm}$, even after sonication. Furthermore, TEM analysis revealed, for all the samples prepared by this technique, the presence of aggregates. Very similar results were obtained for niosomal dispersions obtained with the "proniosome" technique.

Considering the other preparation techniques (TLE-paddle, TLE-vortex, and reverse phase evaporation), the formulations that gave the better results in terms of particle size and PDI, for the series containing Span 60, are presented in Table 1. Rather analogous results were obtained with the corresponding formulations containing Span 40 , even though the use of this surfactant gave generally rise to vesicles of somewhat larger size compared to the corresponding formulations with Span 60 (results not shown).

As reported in Table 1, dynamic light scattering analysis indicated the presence of vesicles with small dimensions (around $200 \mathrm{~nm}$ ) and acceptable homogeneity (PDI 0.27 ) also when Span alone was used. However, the particle size analysis evidenced the presence of a second population of larger particles (around $1 \mu \mathrm{m}$ ), suggesting the presence of aggregation phenomena. TEM analyses confirmed the marked tendency of Span alone niosomes to form aggregates, even at very low surfactant concentration $(0.1 \mathrm{mM})$. On the contrary, this phenomenon did not occur in samples where CHL and SOL were included in the preparation, which did not show any agglomeration phenomena (Figure 1), at least up to a $38 \mathrm{mM}$ total surfactantlipid concentration.

Finally, the "reverse phase evaporation" method allowed for obtaining vesicles of small and homogeneous dimensions (see Table 1); however, the complete removal of chloroform residual from the niosomal suspension was particularly tangled.

\begin{tabular}{|c|c|c|c|c|c|}
\hline Formulation & $\mathrm{Mol} \%$ & $\begin{array}{l}\text { Tot. conc. } \\
(\mathrm{mM})\end{array}$ & $\begin{array}{c}\text { Preparation } \\
\text { method }\end{array}$ & $\begin{array}{c}\text { vesicle size } \\
(\mathrm{nm} \pm \text { S.D.) }\end{array}$ & PDI \\
\hline Span 60 & 100 & 0.1 & TLE-vortex & $243 \pm 12$ & $0.36 \pm 0.03$ \\
\hline Span 60 & 100 & 0.1 & TLE-paddle & $176 \pm 32 *$ & $0.27 \pm 0.03$ \\
\hline Span 60:CHL:SOL & $50: 40: 10$ & 38.0 & TLE-vortex & $316 \pm 87$ & $0.30 \pm 0.03$ \\
\hline Span 60:CHL:SOL & $50: 40: 10$ & 38.0 & Reverse phase ev. & $178 \pm 12 *$ & $0.20 \pm 0.02$ \\
\hline Span 60:CHL:SOL & $50: 40: 10$ & 9.5 & Reverse phase ev. & $222 \pm 10$ & $0.18 \pm 0.03$ \\
\hline Span 60:CHL:SOL & $50: 40: 10$ & 9.5 & TLE-paddle & $233 \pm 20$ & $0.27 \pm 0.02$ \\
\hline
\end{tabular}




\section{Selection of the best experimental conditions for preparing NPG-niosomes}

Based on the results of the previous screening, TLE-paddle and TLE-vortex were selected as preparation methods. The total component concentration was fixed at $38 \mathrm{mM}$, since higher lipid amounts caused problems of excessive viscosity of the samples. Moreover, the SOL amount in the niosomal formulations was set at 10 mol\%, since high levels of SOL are potentially haemolytic (13). The NPG content was fixed at 10 mol\%, because higher amounts resulted in unstable formulations, from which NPG crystallised out within some hours. Therefore, the Span:CHL:SOL:NPG respective amounts were kept constant at the 50:40:10:10 mol\%. The effect of varying the hydration temperature was also evaluated. The mean size and PDI of the obtained formulations are shown in Table 2.

TEM analysis confirmed the results obtained by light-scattering analysis, and demonstrated the actual formation of nano-sized niosomes, as shown for example in Figure 2 for the system obtained using Span 40 as formulation component and the TLE-paddle stirring at $65^{\circ} \mathrm{C}$ as preparation method.

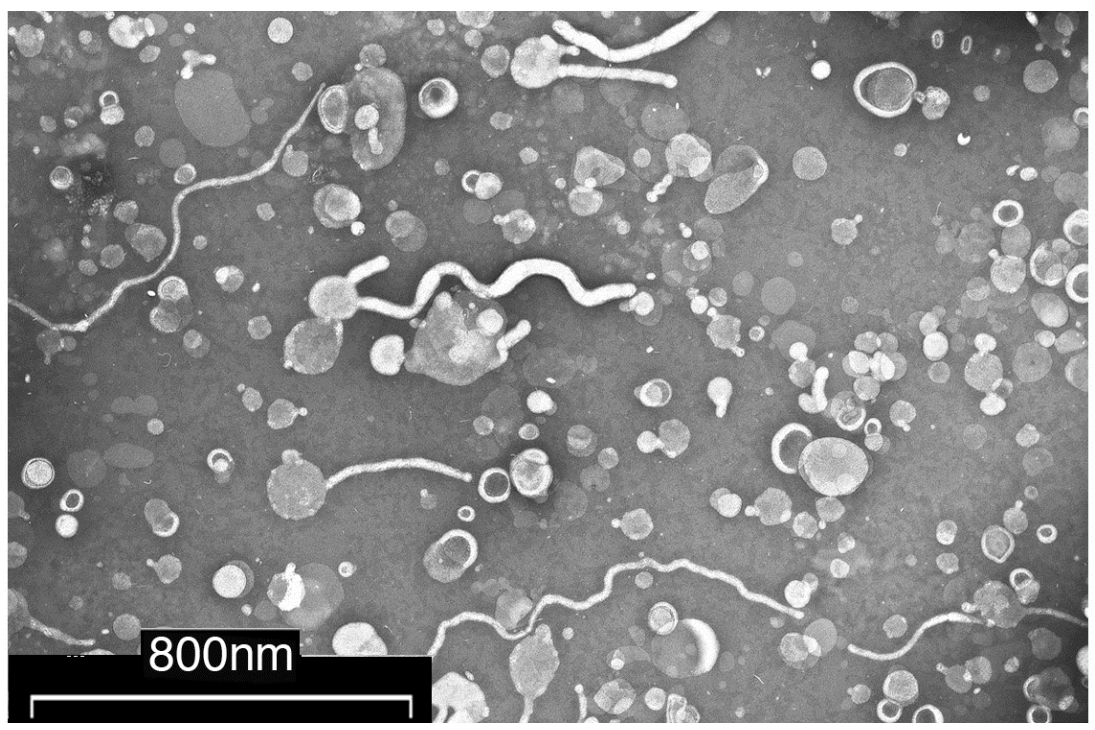

Figure 1. Transmission electron micrograph of Span60:CHL:SOL (50:40:10 mol\%) niosomes obtained by reverse phase evaporation (total surfactant-lipid conc. $9.5 \mathrm{mM}$ ).

Table 2. Effect of formulation composition and preparation method on mean size and polydispersity index (PDI) of NPG-bearing niosomal vesicles $(n=6)$

\begin{tabular}{lcccc}
\hline Formulation & $\begin{array}{c}\text { Preparation } \\
\text { method }\end{array}$ & $\begin{array}{c}\text { Temperature } \\
\left({ }^{\circ} \mathrm{C}\right)\end{array}$ & $\begin{array}{c}\text { vesicle size } \\
(\mathrm{nm} \pm \text { S.D. })\end{array}$ & PDI \\
\hline Span 40:CHL:SOL:NPG & TLE-vortex & 65 & $170 \pm 5$ & $0.25 \pm 0.01$ \\
Span 40:CHL:SOL:NPG & TLE-vortex & 90 & $194 \pm 8^{*}$ & $0.28 \pm 0.03$ \\
Span 40:CHL:SOL:NPG & TLE-paddle & 65 & $152 \pm 5$ & $0.24 \pm 0.01$ \\
Span 40:CHL:SOL:NPG & TLE-paddle & 90 & $185 \pm 7^{*}$ & $0.27 \pm 0.02$ \\
Span 60:CHL:SOL:NPG & TLE-vortex & 65 & $174 \pm 6$ & $0.26 \pm 0.04$ \\
Span 60:CHL:SOL:NPG & TLE-vortex & 90 & $204 \pm 7^{*}$ & $0.33 \pm 0.03$ \\
Span 60:CHL:SOL:NPG & TLE-paddle & 65 & $153 \pm 4$ & $0.25 \pm 0.03$ \\
Span 60:CHL:SOL:NPG & TLE-paddle & 90 & $194 \pm 6^{*}$ & $0.29 \pm 0.02$ \\
\hline (*significantly higher $(\mathrm{P}<0.05)$ than the corresponding value at $\left.65^{\circ} \mathrm{C}\right)$ \\
\hline
\end{tabular}




\section{Preparation and characterization of DOX- loaded NPG-niosomes}

Based on the above results, TLE-paddle method at $65{ }^{\circ} \mathrm{C}$ was then selected for preparing DOXloaded NPG-bearing niosomes, maintaining constant the Span:CHL:SOL:NPG molar ratios (50:40:10:10), and using, alternatively, Span 40 or Span 60. No aggregation phenomena where observed as a consequence of encapsulation of the amphipatic drug DOX, and TEM analysis confirmed the formation of homogeneous nanosized vesicles of red colour.

The mean diameter of drug-loaded vesicles after probe sonication resulted $183 \pm 15 \mathrm{~nm}$ using Span 40 and $161 \pm 4 \mathrm{~nm}$ using Span 60. The PDI was $<0.3$ for both the preparations.

The DOX encapsulation efficiency, indirectly determined by size exclusion chromatography, was $57.8 \% \pm 1.8$ for NPG-niosomes containing Span 60 . On the contrary, surprisingly, it was much lower $(15.0 \% \pm 2.1)$ for those containing
Span 40, which were then excluded from further studies. Entrapment efficiency of DOX-NPGniosomes containing Span 60, indirectly determined by the dialysis technique was $55.0 \pm$ 3.4 , in good agreement with the results obtained by size exclusion chromatography.

The Zeta potential of both drug-loaded and un-loaded NPG-niosomal dispersions was determined at three different $\mathrm{pH}$ values. The results of this study are shown in Table 3 .

\section{Stability studies of NPG-niosomal dispersions}

The stability of both empty and DOX-loaded NPG-niosomal suspensions was monitored in terms of vesicle particle size, PDI, and Zeta potential during six months storage at both 4 and $25{ }^{\circ} \mathrm{C}$, as shown by data reported in Table 4 . Entrapment efficiency of drug loaded NPGniosomal formulations during storage was also checked, following separation of free drug by size exclusion chromatography.

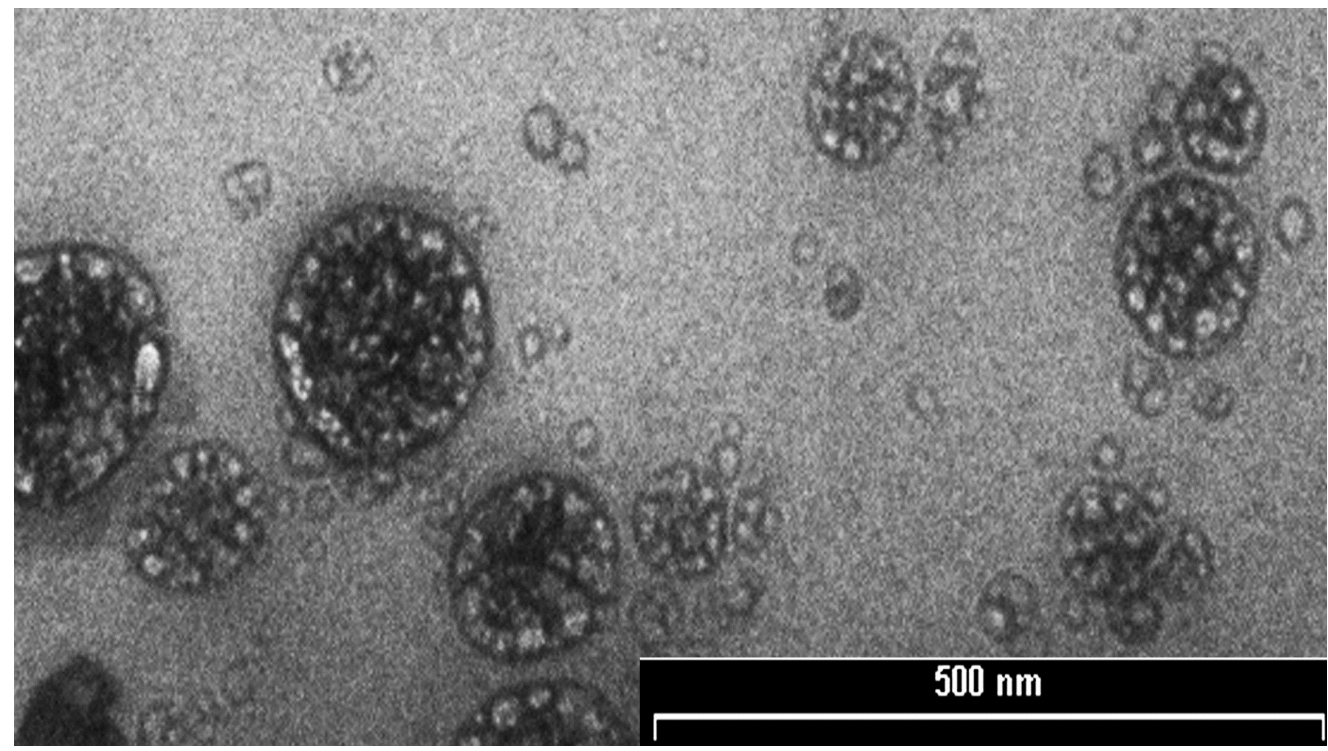

Figure 2. Transmission electron micrograph of Span40:CHL:SOL:NPG (50:40:10:10 molar ratio) niosomes obtained by TLE-paddle method at $65^{\circ} \mathrm{C}$ (total surfactant-lipid conc. $38 \mathrm{mM}$ )

\begin{tabular}{llll}
\hline \multicolumn{4}{l}{ Table 3. Effect of $\mathrm{pH}$ changes on Zeta potential of empty or DOX-loaded NPG-niosomes $(\mathrm{n}=6)$} \\
\hline Sample & $\mathrm{Z}$ pot $(\mathrm{pH}=3)$ & $\mathrm{Z}$ pot $(\mathrm{pH}=5.6)$ & $\mathrm{Z}$ pot $(\mathrm{pH}=9)$ \\
\hline Empty NPG-niosomes & $-1.63 \pm 1.1$ & $-2.05 \pm 1.2$ & $-2.36 \pm 1.3$ \\
DOX-loaded NPG-niosomes & $-1.86 \pm 1.2$ & $-2.29 \pm 1.4$ & $-2.08 \pm 1.1$ \\
\hline
\end{tabular}




\section{In vivo studies}

Table 5 lists the concentrations of DOX in plasma and organs of rats after $60 \mathrm{~min}$ from i.v. injection of $12 \mathrm{mg} / \mathrm{kg}$ of DOX as commercial solution or as NGP-niosomal formulation.

\section{DISCUSSION}

In the present work the possibility of developing a functionalized niosomal formulation based on sorbitan esters (Span) as main surfactant and bearing NPG has been investigated, in view of its future application in active brain delivery of doxorubicin.

Selection of the best preparation method and formulation for niosome preparation

Before proceeding to the preparation of NPGbearing niosomes, a preliminary study was performed for a rational selection of the most effective preparation method and of the best component composition, in order to obtain vesicles with homogeneous and sufficiently small dimensions, suitable for intravenous injection. Moreover, it is known that the size ranges of niosomes have a major effect on their fate in-vivo, and a decrease in vesicle size can significantly reduce their recognition by the complements in the blood and prolong their circulation time in the bloodstream, thus improving the therapeutic activity of the entrapped drugs $(30,31)$.

Based on the results of this initial preliminary phase, proniosome and nitrogen bubbling techniques were excluded from further investigations, since they gave rise to large and poorly homogeneous vesicles, which exhibited a marked tendency to form agglomerates. On the other hand, reverse phase evaporation was discarded, in spite of the suitable dimensions of the obtained vesicles (see Table 2), due to the particular preparation conditions required by this method, which did not allow a complete removal of chloroform residual from the niosomal suspension. Therefore, only TLE-paddle and TLE-vortex preparation methods were selected for the following phase of the study. Sonication was instead chosen as the most effective technique for reducing the vesicle dimensions of niosomal dispersions.

As for the vesicle composition, experimental results clearly evidenced the need of adding to the main surfactant some proper membrane additives, like CHL, a membrane bilayer stabilizer frequently included in niosomal formulations to improve the vesicle physical stability (22), and SOL, a steric stabilizer which provides a steric barrier on the vesicle surface (32), thus preventing the formation of aggregates.

Table 4. Effect of six months storage at $4^{\circ} \mathrm{C}$ or room temperature $\left(25^{\circ} \mathrm{C}\right)$ on size, polydispersity index (PDI), Zeta potential and entrapment efficiency (EE\%) of empty and DOX-loaded NPG-niosomes containing Span 60:CHL:SOL:NPG (50:40:10:10 molar ratio)

\begin{tabular}{lcccccccc}
\hline Sample & \multicolumn{2}{c}{ Size $(\mathrm{nm})(\mathrm{n}=6)$} & \multicolumn{2}{c}{ PDI $(\mathrm{n}=6)$} & \multicolumn{2}{c}{ Z Potential $(\mathrm{n}=6)$} & \multicolumn{2}{c}{ EE\% $(\mathrm{n}=5)$} \\
\cline { 2 - 9 } & day 0 & day 360 & day 0 & day 360 & day 0 & day 360 & day 0 & day 360 \\
\hline Empty, $4{ }^{\circ} \mathrm{C}$ & $153 \pm 4$ & $155 \pm 5$ & $0.20 \pm 0.04$ & $0.22 \pm 0.02$ & $-1.7 \pm 1.3$ & $-2.1 \pm 1.2$ & -- & -- \\
Empty, $25^{\circ} \mathrm{C}$ & $153 \pm 4$ & $162 \pm 8$ & $0.20 \pm 0.04$ & $0.23 \pm 0.04$ & $-1.7 \pm 1.3$ & $-1.4 \pm 0.9$ & -- & - \\
Loaded, $4{ }^{\circ} \mathrm{C}$ & $161 \pm 4$ & $166 \pm 7$ & $0.23 \pm 0.02$ & $0.23 \pm 0.02$ & $-1.9 \pm 1.2$ & $-1.8 \pm 1.2$ & $57.8 \pm 1.8$ & $55.9 \pm 3.2$ \\
Loaded, $25^{\circ} \mathrm{C}$ & $161 \pm 4$ & $169 \pm 7$ & $0.23 \pm 0.02$ & $0.24 \pm 0.03$ & $-1.9 \pm 1.2$ & $-1.7 \pm 1.2$ & $57.8 \pm 1.8$ & $53.9 \pm 3.2$ \\
\hline
\end{tabular}

Table 5. Doxorubicin (DOX) concentrations $(\mu \mathrm{g} / \mathrm{g})$ in plasma and organs of rats after 60 min from i.v. injection of $12 \mathrm{mg} \mathrm{DOX} / \mathrm{kg}$ as commercial solution (Ebewe, $2 \mathrm{mg} / \mathrm{mL}$ ) or NGP-niosomal formulation

\begin{tabular}{lccccc}
\hline Sample & plasma & heart & brain & liver & kidneys \\
\hline commercial DOX & $0.5 \pm 0.1$ & $1.3 \pm 0.2$ & $<0.1$ & $14.5 \pm 1.6$ & $42.3 \pm 4.8$ \\
NPG-niosomal DOX & $1.0 \pm 0.2^{*}$ & $0.4 \pm 0.1^{*}$ & $2.9 \pm 0.4^{*}$ & $17.1 \pm 1.9$ & $38.2 \pm 4.6$ \\
\hline
\end{tabular}

(*significantly different $(\mathrm{P}<0.05)$ from the corresponding value of the commercial sample) 
The use of the TLE-paddle method instead of the TLE-vortex method did not significantly affect the niosome mean particle size $(\mathrm{P}>0.05)$. However, the TLE-paddle technique generally allowed for obtaining vesicles with smaller size and better homogeneity. Analogous findings were also obtained by using Span 40 rather than Span 60 in niosomal formulations, and, also in this case, the observed differences in vesicle particle size were not statistically significant $(\mathrm{P}>0.05)$. Both these findings indicated the good robustness of the selected niosomal vesicle formulation (Span:CHL:SOL:NPG 50:40:10:10 molar ratio), whose mean size and homogeneity were statistically unaffected $(\mathrm{P}>0.05)$ by changing the preparation method (TLE-paddle or TLE-vortex) and/or the surfactant type (Span 60 or 40).

The possible benefits obtained by using higher hydration temperatures during the vesicle preparation were also evaluated. However, differently from that reported by other authors $(9$, 10), the temperature increase did not improve the properties of the vesicles in terms of either reduction in size or increase in homogeneity. On the contrary, in most cases the vesicle dimensions of niosomal dispersions prepared at $90^{\circ} \mathrm{C}$ resulted greater than those of the corresponding systems prepared at $65^{\circ} \mathrm{C}$. Therefore, the temperature of $65^{\circ} \mathrm{C}$ was selected for the last part of the study, devoted to the preparation of NPGbearing niosomes loaded with DOX. The choice of the lower preparation temperature was also strengthened by results of DOX stability studies, which showed a partial degradation of the drug after $1 \mathrm{~h}$ stirring of its aqueous solution at $90^{\circ} \mathrm{C}$, where only the $85.6 \%$ of the drug remained unaltered. On the contrary, no degradation was detected by HPLC analysis of the corresponding sample kept in the same conditions at $65^{\circ} \mathrm{C}$.

\section{Preparation and characterization of DOX- loaded NPG-niosomes}

The developed formulation allowed for preparing DOX-loaded vesicles with homogeneous nanosized dimensions. However, a satisfying encapsulation efficiency, near to $60 \%$, was obtained only when using Span 60. A possible explanation of the higher entrapment efficiency of formulations containing Span 60 than Span 40 could be the longer saturated alkyl chain of the first, which confers less permeability to the vesicle membrane (33). However, the reasons for this unexpected result need to be further addressed.
In order to get some insight into the modality of drug entrapment into the niosomal vesicles, the effect of $\mathrm{pH}$ variations on Zeta potential of both drug-loaded and un-loaded niosomal dispersions was determined. The Zeta potential of empty NPG-niosomes did not change in important way by varying $\mathrm{pH}$ from 3 to 9 , indicating the absence of ionization phenomena of the membrane components (see Table 3). Interestingly, drug loading did not significantly change $(\mathrm{P}>0.05)$ the Zeta potential of NPG-niosomes with respect to the un-loaded ones. This finding seems to indicate that DOX should be actually entrapped into the vesicle core and not simply inserted in the membrane bilayer. In fact, it has been shown that DOX loading significantly influences the Zeta potential of the vesicles when it is inserted in the vesicle membrane $(22,34)$, since, being a weak base $(\mathrm{pKa}=8.3)$, it is differently ionized as a function of $\mathrm{pH}$.

Both unloaded and drug-loaded NPGniosomes demonstrated good stability over the storage period considered (Table 4). In fact, no statistically significant variations in size and Zeta potential values of the empty vesicle were observed $(\mathrm{P}>0.05)$. Analogous results were obtained for DOX-loaded NPG-niosomes. Moreover, the reduction of drug encapsulation efficiency of this system after six months storage at ambient temperature was not significant $(\mathrm{P}>0.05)$ with respect to the freshly prepared sample.

\section{In vivo studies}

The developed DOX NPG-niosomal formulation was injected i.v. in rats, in comparison with a commercial solution formulation (Ebewe, Sandoz), in order to evaluate its actual effectiveness in enhancing DOX brain delivery. No plain DOX niosomes were tested as control in this preliminary in vivo study, with the intention of limiting as much as possible the number of animals to be sacrificed. This choice was made also on the basis of the studies of Dufes et al. (10), which already demonstrated the significantly superior ability of NPG-niosomes than control vesicles in increasing the brain uptake of the vasoactive intestinal peptide (VIP).

Significantly higher $(\mathrm{P}<0.05)$ DOX plasma levels were obtained with the NPG-niosomal dispersion with respect to the commercial solution at the same drug dosage, indicating that the developed formulation was able to keep the drug longer in the blood circulation (Table 5). No significant differences $(\mathrm{P}>0.05)$ between the two 
formulations were instead observed in the respective drug levels in liver and kidneys. On the contrary, NPG-niosomal formulation reduced the accumulation of drug in the heart with respect to the DOX commercial solution, giving rise to significantly lower $(\mathrm{P}<0.05)$ DOX levels in this organ. Finally, even more important, the DOX concentration achieved in the brain after $60 \mathrm{~min}$ from i.v. administration of the NPG-niosomal formulation was $2.9 \pm 0.4 \mu \mathrm{g} / \mathrm{g}$. On the contrary, i.v. administration of DOX commercial solution yielded brain concentrations of the drug below the detection limit $(<0.1 \mu \mathrm{g} / \mathrm{g}) \quad$ (Table 5). These results indicated that the glucose-targeted vesicles were actually able to significantly improve the brain delivery of DOX, confirming the results obtained for the vasoactive intestinal peptide (10). The exact mechanism of this transport so far is not known. It has been hypothesized that the glucose ligands present on the vesicles surface could be recognized by the cells of BBB, which express high levels of the glucose transporters GLUT1, thus allowing an efficient brain uptake and drug delivery in the brain (10). An additional mechanism could be the improved retention of the vesicles in the brain blood capillaries joined with an adsorption to the capillary wall, both concurring to give rise to a higher concentration gradient that would enhance the drug transport across the endothelial cell layer (35). A possible surfactant effect could be also considered, characterized by a solubilisation of the endothelial cell membrane lipids, leading to membrane fluidization and increased drug permeation through the BBB (35). A further possibility could be the endocytosis of vesicles by the endothelial cells, followed by the delivery of the drug within these cells and then delivery to the brain (36). All of these mechanisms also could operate in combination.

\section{CONCLUSIONS}

The study allowed for developing a nano-sized, stable and safe NPG-bearing niosomal formulation able to effectively encapsulate DOX. The developed formulation, in virtue of its relatively high efficiency of drug encapsulation and good stability, could find useful applications as an effective tool for achieving DOX brain delivery, exploiting the brain uptake properties of the glucose-targeted vesicles (10).

Preliminary in vivo studies showed that i.v. administration of a single dose of the developed NPG-niosomal formulation with respect to the commercial one was able not only to significantly reduce the hearth accumulation of the drug and to keep longer it in the blood circulation, but, above all, to allow the achievement of well detectable DOX brain concentrations. Therefore, the use of glucose-bearing niosomes seems to represent a novel useful tool to improve DOX delivery in the brain in the treatment of brain tumours, further supported by the fact that GLUT-1 receptors are over expressed in solid tumours.

The encouraging and promising results obtained in this initial in vivo study warrant future experiments aimed at determining the main pharmacokinetic parameters of NPG-niosomal DOX and gaining more insight about the actual mechanism of the enhanced DOX brain delivery achieved by the niosomal formulation. In the ambit of these studies, administration of DOX not-decorated niosomes as well as of DOX solution mixed with empty NPG-niosomes will be helpful to elucidate the reasons for the obtained results. Furthermore, future studies will be also necessary for improving the performance of the developed formulation and obtaining a most efficient drug brain delivery. Considering the high versatility of the niosomal formulation, different approaches will be taken into account, such as the inclusion in the vesicle membrane of positively charged components, to further improve the brain cellular uptake (37), or the inclusion of DOX together with an inhibitor of the cellular efflux pumps (38).

\section{REFERENCES}

1. Jeanneret LJ. The targeted delivery of cancer drugs across the blood-brain barrier: chemical modifications of drugs or drug-nanoparticles? Drug Discovery Today 2008; 13: 1099-1105.

2. Misra A, Ganesh S, Shahiwala A, Shah SP. Drug delivery to the central nervous system: a review. J. Pharm. Pharmaceut. Sci. 2003; 6: 252-273.

3. Patel MM, Goval BR, Bhadada SV, Bhatt JS, Amin AF. Getting into the brain: approaches to enhance brain drug delivery. CNS Drugs 2009; 23: 35-58.

4. Kreuter J. Nanoparticulate systems for brain delivery of drugs, Adv. Drug Deliv. Rev. 2001; 47: 65-81.

5. Koziara JM, Lockman PR, Allen DD, Mumper RJ. The blood-brain barrier and brain drug delivery. J. Nanosci. Nanotechnol. 2006; 6: 2712-2735.

6. Fang JY, Hong CT, Chiu WT, Wang YY, Effect of liposomes and niosomes on skin permeation of enoxacin. Int. J. Pharm. 2001; 
219: 61-72.

7. Attia IA, El-Gizawy SA, Fouda MA, Donia AM. Influence of a Niosomal Formulation on the Oral Bioavailability of Acyclovir in Rabbits AAPS PharmSciTech. 2007; 8: 206-212

8. Yadav JD, Kulkarni PR, Vaidya KA, Shelke GT. Niosomes: a review. J. Pharm. Res. 2011; 4: 632-636.

9. Dufes C, Schatzlein AG, Tetley L, Gray AL, Watson DG, Olivier JC, Uchegbu IF. Niosomes and Polymeric Chitosan Based Vesicles Bearing Transferrin and Glucose Ligands for Drug Targeting. Pharm. Res. 2000; 17: 1250-1258.

10. Dufes C, Gaillard F, Uchegbu IF, Schatzlein AG, Olivier JC, Muller JM. Glucose-targeted niosomes deliver vasoactive intestinal peptide (VIP) to the brain. Int. J. Pharm. 2004; 285: 7785.

11. Stan AC, Casares S, Radu D, Walter GF, Brumeanu TD. Doxorubicin-induced cell death in highly invasive human gliomas. Anticancer Res. 1999; 19: 941-950.

12. Uchegbu IF, Double JA, Turton JA, Florence AT. Distribution, metabolism and tumoricidal activity of doxorubicin administered in sorbitan monostearate (Span-60) niosomes in the mouse. Pharm. Res. 1995; 12: 1019-1024.

13. Uchegbu IF, Duncan R. Niosomes containing $N$-(2-hydroxypropyl)methacrylamide copolymer-doxorubicin (PK1): effect of method of preparation and choice of surfactant on niosome characteristics and a preliminary study of body distribution. Int. J. Pharm. 1997; 155: 7-17.

14. Charrois GJR, Allen TM. Drug release rate influences the pharmacokinetics, biodistribution, therapeutic activity, and toxicity of pegylated liposomal doxorubicin formulations in murine breast cancer. Biochim. Biophys. Acta 2004; 1663: 167-177.

15. Fritze A, Hens F, Kimpfler A, Schubert R, Peschka-Suiss R. Remote loading of doxorubicin into liposomes driven by a transmembrane phosphate gradient. Biochim. Biophys. Acta 2006; 1758: 1633-1640.

16. Li X, Ding L, Xu Y, Wang Y, Ping Q. Targeted delivery of doxorubicin using stealth liposomes modified with transferrin. Int. J. Pharm. 2009; 373: 116-123.

17. Dufes C, Muller JM, Couet W., Olivier JC, Uchegbu IF, Schatzlein AG. Anticancer drug delivery with transferring targeted polymeric vesicles. Pharm. Res. 2004; 21: 101-107.

18. Gulyaev AE, Gelperina SE, Skidan IN, Antropov AS, Kivman GY, Kreuter J. Significant transport of doxorubicin into the brain with polysorbate80-coated nanoparticles. Pharm. Res. 1999; 16: 1564-1569.

19. Gil ES, Li J, Xiao H, Lowe TL. Quaternary ammonium ß-cyclodextrin nanoparticles for enhancing doxorubicin permeability across the in vitro blood-brain barrier. Biomacromolecules 2009; 10: 505-516

20. Nishioka T, Oda Y, Seino Y, Yamamoto T, Inagaki $\mathrm{N}$, Yano $\mathrm{H}$, Imura $\mathrm{H}$, Shigemoto $\mathrm{R}$, Kikuchi H. Distribution of the Glucose Transporters in Human Brain Tumours. Cancer Res. 1992; 52: 3972-3979.

21. Smith TAD. Facilitative glucose transporter expression in human cancer tissue. Br. J. Biomed. Sci. 1999; 56: 285-292.

22. Uchegbu IF, Vyas SP. Non-ionic surfactant based vesicles (niosomes) in drug delivery. Int. J. Pharm. 1998; 172: 33-70.

23. Yoshioka T, Sternberg B, Florence AT. Preparation and properties of vesicles (niosomes) of sorbitan monoesters (Span 20, 40, 60 and 80) and a sorbitan triester (Span 85). Int. J. Pharm. 1994; 105: 1-6.

24. $\mathrm{Hu} \mathrm{C}$, Rhodes DG, Proniosomes: a novel drug carrier preparation. Int. J. Pharm. 1999; 185, 2335.

25. Kobayashi $\mathrm{T}$, Ishida $\mathrm{T}$, Okada $\mathrm{Y}$, Ise $\mathrm{S}$, Harashima H, Kiwada H. Effect of transferrin receptor-targeted liposomal doxorubicin in $\mathrm{P}$ glycoprotein-mediated drug resistant tumour cells. Int. J. Pharm. 2007; 329: 94-102.

26. Shen Y, Tu J. Preparation and ocular pharmacokinetics of ganciclovir liposomes. AAPS Journal 2007; 9: 371-377.

27. Tavano L, Muzzalupo R, Trombino S, Cassano R, Pingitore A, Picci N. Effect of formulation variables in the in vitro percutaneous permeation of sodium diclofenac from new vesicular systems obtained from Pluronic triblock copolymers. Colloids and Surfaces B: Biointerfaces 2010; 79: 227-234.

28. Bragagni M, Maestrelli F, Mennini N, Ghelardini C, Mura P. Liposomal formulations of prilocaine: effect of complexation with hydroxypropyl- $\beta$-cyclodextrin on drug anaesthetic efficacy. J. Lipos. Res. 2010; 20: 315-332.

29. Cielecka-Pionteka J, Jelinskaa A, Zajac M, Sobczak M, Bartold A, Oszczapowicz I. A comparison of the stability of doxorubicin and daunorubicin in solid state. J. Pharm. Biomed. Anal. 2009; 50: 570-579.

30. Harashima H, Hiraiwa T, Ochi Y, Kiwada H. Size-dependent liposome degradation in blood: in vitro/in vivo correlation by kinetic modelling. J. Drug Target. 1995; 3: 253-261.

31. Nagayasua A, Uchiyamab K, Kiwadab H. The size of liposomes: a factor which affects their targeting efficiency to tumours and therapeutic activity of liposomal antitumor drugs. Adv. Drug Deliv. Rev. 1999; 40: 75-87.

32. Arunothayanun P, Bernard MS, Craig DQM, Uchegbu IF, Florence AT. The effect of processing variables on the physical characteristics of non-ionic surfactant vesicles 
(niosomes) formed from a hexadecyl diglycerol ether. Int. J. Pharm. 2000; 201: 7-14.

33. Hao Y, Zhao F, Yanhong NL, Li K. Studies on high encapsulation of colchicine by a niosome system. Int. J. Pharm. 2002; 244: 73-80.

34. Sahin NO. Niosomes as carrier systems, in: Mozafari MR (ed.), Nanomaterials and nanosystems for Biomedical Applications, Springer, Dordrecht, The Netherlands. 2007, pp 67-82.

35. Kreuter J. Influence of the surfactant properties on nanoparticle-mediated transport of drugs to the brain. J. Nanosci. Nanotech. 2004; 4: 484488.
36. Kreuter J, Shamenkov D, Petrov V, Ramge P, Cychutek K, Koch-Brandt C, Alyautdin R. Apolipoprotein-mediated transport of nanoparticle-bound drugs across the blood-brain barrier, J. Drug Target. 2002; 10: 317-325.

37. Begley DJ. Delivery of therapeutic agents to the central nervous system: the problems and possibilities. Pharmacol. Therap. 2004; 104: 2945.

38. Rousselle C, Clair P, Lefacounnier J, Kaczorek M, Schermann JM, Temsaman JM. New advances in the transport of doxorubicin through the blood-brain barrier by a peptide vector-mediated strategy. Mol. Pharmacol. 2000; 57: 679-686. 